\title{
Isolated complete plantar dislocation of first metatarsocuneiform (first tarsometatarsal) joint: A case report
}

\author{
by Asif Sultan ${ }^{1}$, Mohd Iqbal Wani ${ }^{1}$, Tahir Ahmad Dar ${ }^{1}$, Mubashir Maqbool Wani ${ }^{1}$, Samina Shafi ${ }^{2}$
}

The Foot and Ankle Online Journal 3 (12): 2

Isolated plantar dislocation of first metatarsocuneiform joint is a rare occurrence and only few have been reported till date to the best of our knowledge. We report such a case of traumatic isolated pure plantar dislocation of first metatarsocuneiform joint in a young otherwise normal patient who was managed by closed reduction. No complication was found and he returned to his pre injury status in 12 weeks with no sequelae found at 2 year follow up.

Key words: Metatarsocuneiform joint, plantar dislocation, tarsometatarsal joint, closed reduction.

This is an Open Access article distributed under the terms of the Creative Commons Attribution License. It permits unrestricted use, distribution, and reproduction in any medium, provided the original work is properly cited. @The Foot and Ankle Online Journal (www.faoj.org)

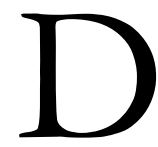
islocations of first metatarsocuneiform joint have been reported with addition of atypical medial, lateral and floating dislocations by several authors ${ }^{1-6}$ usually in association with fractures of metatarsals or dislocations of other tarsometatarsal joints. However, the isolated plantar dislocation of the same joint is of an extremely rare occurrence. The authors present a case of traumatic isolated plantar dislocation of first metatarsocuneiform joint in a young otherwise normal patient who was managed by closed reduction.

Address correspondence to: Dr. Asif Sultan Government hospital for bone and Joint Surgery Barzullah, Srinagar India 190005.

Email;drasifsultan@yahoo.com

\footnotetext{
${ }^{1}$ Government hospital for bone and Joint Surgery Barzullah, SrinagarIndia 190005.

${ }^{2}$ Resident Government Medical College Srinagar.
}

\section{Case Report}

We received a case in our emergency room who had suffered trauma right foot. The patient complained of severe pain and inability to bear weight. The patient was a 22 year-old male Asian Indian in origin. The cause of the trauma was a 5 kilogram rounded iron weight (used for weighing different things in stores across rural India) which had fallen straight on the dorsum of his right foot. On examination there was an obvious deformity of the midfoot medially around first metatarsocuneiform (tarsometatarsal) junction. There was minimal swelling, but tenderness at first tarsometatarsal joint area. The dorsalis pedis pulsations were normal palpable and sensations of foot were normal. There were no signs of compartment syndrome of foot. There was no joint hyperlaxity associated. 

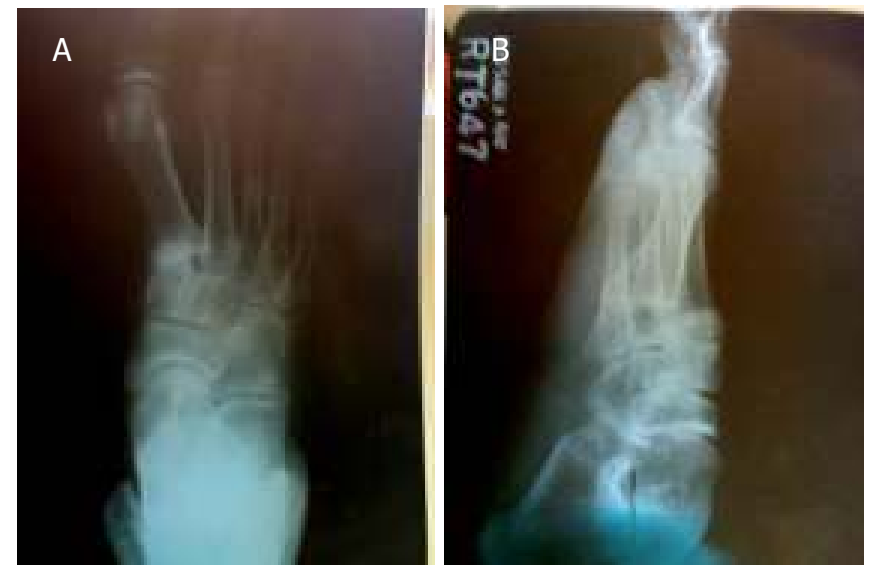

Figure 1A and 1B Anteroposterior (A) and Lateral (B) radiograph showing first metatarsocuneiform dislocation.

Radiographs of the right foot were performed and the anteroposterior view and a lateral view showed planter dislocation of first metatarsocuneiform joint without any associated fracture of metatarsals or cuneiform. (Figs. 1A and 1B) The patient was taken to the operation theatre where under regional anaesthesia (ankle block) the dislocation was reduced anatomically and stability confirmed under C-arm and a short leg cast was given for 6 weeks. Partial weight bearing was started at 6 weeks progressing to full unsupported weight bearing at 12 weeks. The patient had an uneventful recovery and was asymptomatic at two year follow up with normal radiographs, a normal gait with well maintained medial longitudinal arch of foot. (Figs. 2A and 2B)

\section{Discussion}

Dislocation of first metatarsocuineoform joint is a rare injury and it is usually associated with injuries to other tarsometatarsal joints including fractures and dislocations (Lisfranc's fracture dislocation). These injuries may be caused by direct or indirect trauma, varying from low impact injuries such as twisting, to high impact injuries such as road traffic accidents and fall from height. Dislocation of metatarsal is primarily dorsal in indirect trauma with secondary displacement either medially or laterally.
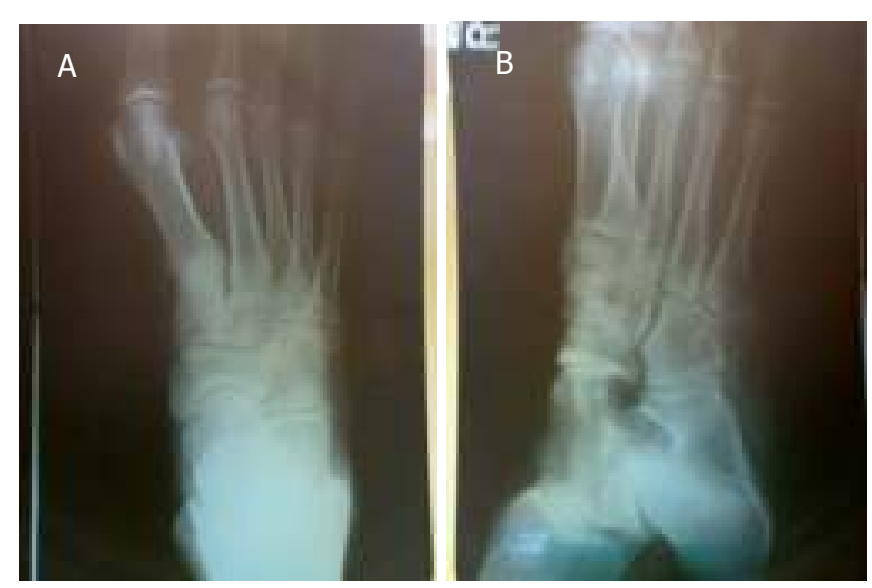

Figure 2A and 2B Anteroposterior (A) and Lateral (B) radiograph showing reduction of first metatarsocuneiform dislocation after 2 years.

This is seen when the foot is planter flexed with weight borne by metatarsal heads, compressing the foot in longitudinal axis causing it to bow (hyper planter flexion) rupturing capsule and ligaments of tarsometatarsal joint resulting in dorsal dislocation. Also the strong structures of the sole of foot, such as the planter fascia and intrinsic muscles reinforce the planter surface of this joint and thus resist dorsiflexion of the metatarsals and planter dislocation of the tarsometatarsal joint. ${ }^{7,8,9}$

Dislocations due to direct trauma require tremendous force such as run over by a heavy object or struck by a falling weight. The metatarsals are displaced plantarward with secondary displacement medially or laterally, depending on the line of force. ${ }^{7,89}$ In our case the falling weight had struck directly over the base of first metatarsal resulting in the planter dislocation at metatarsomedial cuneiform joint without any associated fracture.

Most studies recommend open reduction and fixation with screws or $\mathrm{K}$ wires ${ }^{6,9,10,11}$ in complex fracture dislocations of tarsometatarsal joints. The important determinant of outcome in the treatment of injuries of tarsometatarsal joint is accurate anatomic reduction. ${ }^{10}$ Our patient was managed conservatively by closed reduction achieving anatomic reduction, which was found stable and short leg cast with an uneventful recovery. 


\section{Conclusion}

A high index of suspicion should be maintained to rule out tarsometatarsal joint injuries when examining a patient with an injured foot because delayed or missed diagnosis occurs in up to $20 \%$ of cases. ${ }^{10}$ Left untreated, such disruption may result in marked disability characterized by painful posttraumatic arthritis and deformity ${ }^{11}$ with the goal of treatment being restoration of pain free and a functional foot.

\section{References}

1. Charrois O, Begue T, Muller GP, Masquelet AC. Plantar dislocation of the tarso-metatarsal articulation (Lisfranc articulation). Apropos of a case. Rev Chir Orthop.1998; 84:197201.

2. Mehara AK, Bhan S. Isolated fracture-dislocations of the first tarsometatarsal joint. J Trauma. 1992 Nov; 33(5):683-686.

3. Milankov M, Miljkovic N, Popovic N. Concomitant plantar tarsometatarsal (Lisfranc) and metatarsophalangeal joint dislocations. Arch Orthop Trauma Surg 2003; 123: 95-97.

4. Cuenca Espierrez J, Martinez AA, Herrera A, Panisello JJ. The floating metatarsal: first metatarsophalangeal joint dislocation with associated Lisfranc dislocation. J Foot Ankle Surg. 2003; 42(5):309-311.

5. Shetty MS, Pinto D, Bhardwaj P. Isolated floating first metatarsal: report of an unusual injury. J Foot Ankle Surg. 2007 May-Jun; 46(3):185-187.

6. García Mata S, Hidalgo Ovejero A, Martinez Grande M. Complete dorsal tarsometatarsal dislocation of the first metatarsal. Arch Orthop Trauma Surg 2003; 123: 95-97.

7. Wilson DW. Injuries of the tarsometatarsal joint: etiology, classification and results of treatment. JBJS1972; 64B:677-668.

8. Aitken AP, Poulson D. Dislocations of the tarsometatarsal joint. JBJS 1963; 45A: 246-260.

9. Arntz CT, Hansen ST jr. Dislocations and fracture dislocations of the tarsometatarsal joints. Orthop Clinics North America 1987; 18(1):105-114.

10. Gossens M, De Stoop n. Lisfranc's fracture dislocations: etiology,radiology, and results of treatment. A review of 20 cases. Clin Orthop 1983;176:154-162.

11. Thompson MC, Mormino MA. Injury to the Tarsometatarsal Joint Complex.

J Am Acad Orthop Surg. 2003; 11:260-267. 